\title{
Phase Equilibrium and Diffusion of Solvents in Polybutadiene: A Capillary-Column Inverse Gas Chromatography Study
}

\author{
W. D. CAI, ${ }^{1}$ N. RAMESH, ${ }^{1}$ F. TIHMINLIOGLU, ${ }^{2}$ R. P. DANNER ${ }^{1}$, J. L. DUDA, ${ }^{1}$ A. De HAAN $^{3}$ \\ ${ }^{1}$ Center for the Study of Polymer-Solvent Systems, Department of Chemical Engineering, The Pennsylvania State \\ University, University Park, Pennsylvania 16802 \\ ${ }^{2}$ Izmir Institute of Technology, Chemical Engineering Department, Gulbahce 35437, Urla-Izmir, Turkey \\ ${ }^{3}$ University of Twente, Faculty of Chemical Technology, PO Box 217, 7500 AE Enschede, The Netherlands
}

Received 6 August 2001; revised 12 February 2002; accepted 13 February 2002

\begin{abstract}
The capillary-column inverse gas chromatography method was used to measure the diffusion and partition coefficients of ethylbenzene, styrene, and acrylonitrile in polybutadiene (PBD) at infinite dilution of the solvents. Experiments were performed over a temperature range of $50-125{ }^{\circ} \mathrm{C}$. At temperatures well above the glass-transition temperature of PBD, the diffusivities were correlated using an Arrhenius expression. The Arrhenius parameters in turn were intercorrelated and shown to be a function of the occupied volume, thus providing a method for predicting the diffusion of other solvents in the same polymer. Further, the activation energy was predicted using the Duda-Vrentas free-volume approach. The activation energy thus obtained was compared with the activation energy of the Arrhenius approach. The weight-fraction activity coefficient data were compared to the predictions of the group contribution, lattice-fluid equation-of-state, and the UNIquac Functional-group Activity Coefficient (UNIFAC) free-volume models. (C) 2002 Wiley Periodicals, Inc. J Polym Sci Part B: Polym Phys 40: 1046-1055, 2002

Keywords: inverse gas chromatography; polybutadiene; ethylbenzene; styrene; acrylonitrile; diffusion; phase behavior; UNIFAC; lattice fluid; equation of state; freevolume theory
\end{abstract}

\section{INTRODUCTION}

Information on the migration and partitioning of small molecules in polymeric materials is important in many practical applications-drying of films, paints, or coatings; devolatilization of bulk particles; extrusion; foam production; membrane separations; and so forth. To investigate the

Correspondence to: R. P. Danner (E-mail: rpd@psu.edu) Journal of Polymer Science: Part B: Polymer Physics, Vol. 40, 1046-1055 (2002) ๑ 2002 Wiley Periodicals, Inc. transport and thermodynamic behaviors of these systems, the solubility and diffusivity of the low molecular weight components of a polymer solution are the required fundamental properties.

Capillary-column inverse gas chromatography (CCIGC) has been used by a number of researchers to determine the partition and diffusion coefficients in polymeric systems. ${ }^{1-3}$ The principle behind this technique is based on the distribution of volatile solvent molecules between a mobile gas phase and a stationary polymeric phase. Inverse gas chromatography (IGC) is an efficient, reliable, 
and accurate technique for these measurements, particularly at the limit of infinite dilution.

The free-volume theory of Vrentas and Duda ${ }^{4,5}$ is frequently used to correlate and predict temperature- and concentration-dependent diffusion on the basis of data at temperatures close to the glass-transition temperature $\left(T_{\mathrm{g}}\right)$. Solvent diffusion in polymers with trace amounts of solvent far above the $T_{\mathrm{g}}$ of the polymer, on the other hand, are typically correlated using the Arrhenius approach. The Arrhenius approach uses a constant activation energy and pre-exponential term to correlate and predict diffusivity. At temperatures far above the $T_{\mathrm{g}}$, a plot of the $\log$ of diffusivity versus reciprocal absolute temperature is linear.

The object of this work was to conduct a detailed study of the diffusion and solubility of ethylbenzene, styrene, and acrylonitrile in polybutadiene (PBD). PBD is the homopolymer of butadiene that is widely used in tire tread and sidewall compounds and is an important impact modifier in high-impact polystyrene and acrylonitrile-butadiene-styrene resins. Other researchers have reported diffusion data for different solvents in PBD; however, most studies do not measure infinite dilution coefficients directly but extrapolate from finite concentration data. ${ }^{6-10}$ In this study, the partition coefficient at infinite dilution was compared with the predictions of group contribution, lattice-fluid equation-of-state, and UNIFAC freevolume theories. The possibility of using diffusivity to predict diffusion of other solvents in the same polymer was investigated. All the IGC experiments were conducted well above the $T_{\mathrm{g}}$ of the polymer where free volume does not limit transport.

\section{THEORY}

\section{CCIGC}

Invoking the continuity equations for the solvent in the gas and polymer phases and appropriate initial and boundary conditions, Pawlisch et al. ${ }^{11}$ developed a method to determine solubility and diffusivity in polymer-solvent systems. This analysis involves three dimensionless groups $-\alpha, \beta$, and $\gamma$

$$
\alpha=\frac{R}{K \tau} \quad \beta^{2}=\frac{\tau^{2} u}{D_{\mathrm{p}} L}
$$

where $\alpha$ and $\beta$ are related to the solubility and diffusivity of the solvent in the polymer-solvent system. The third dimensionless group, $\gamma$, is re- lated to the gas-phase diffusivity, gas velocity, and column length that are inputs to the model

$$
\gamma=\frac{D_{\mathrm{g}}}{u L}
$$

where $L$ is the length of the column, $u$ is the mean velocity of the carrier gas, $R$ is the radius of the gas-polymer interface, $D_{\mathrm{g}}$ and $D_{\mathrm{p}}$ are the gasphase and stationary-phase diffusion coefficients for the solute, $\tau$ is the thickness of the polymer coating in the capillary column, and $K$ is the equilibrium partition coefficient relating the gasphase concentration to the polymer-phase concentration.

\section{Correlation of the Diffusion Coefficients}

The PBD has a $T_{\mathrm{g}}$ of $-95^{\circ} \mathrm{C}$. Because the lowest temperature at which data were collected in this study was $20{ }^{\circ} \mathrm{C}$, the polymer was always well above the $T_{\mathrm{g}}$ and hence in the rubbery state. In these cases the diffusion behavior can usually be described by the following Arrhenius expression:

$$
\ln \left(D_{\mathrm{p}}\right)=\ln \left(D_{\mathrm{o}}\right)-\frac{E_{\text {Arr }}}{R_{\mathrm{g}} T}
$$

In the aforementioned equation, $D_{o}$ is the preexponential factor, whereas $E$ is the activation energy for diffusion. The diffusivity of a trace amount of solvent in a polymer according to the free-volume theory is given by ${ }^{4,5}$

$$
\ln \left(D_{\mathrm{p}}\right)=\ln \left(\tilde{D}_{\mathrm{o}}\right)-\frac{\gamma \hat{V}_{2}^{*} \xi}{K_{12}\left(K_{22}-T_{\mathrm{g}^{2}}+T\right)}
$$

In eq $3, \tilde{D}_{\mathrm{o}}$ is the pre-exponential term used in the free-volume theory, and $T_{\mathrm{g} 2}$ is the glass-transition temperature of the pure polymer. $K_{12} / \gamma$ and $K_{22}$ are free-volume parameters of the polymer obtained from correlating viscosity-temperature data with the Williams-Landel-Ferry equation. In addition, $\xi$ is the ratio of the molar volume of the jumping unit of the solvent to that of the polymer. The pre-exponential terms in the Arrhenius approach, $D_{\mathrm{o}}$ (eq 3 ), and in the free-volume theory, $\tilde{D}_{\text {o }}$ (eq 4 ) are different. Equation 4 also neglects the energy for solvent molecules to move away from its neighbors. This is a good approximation for most systems but could be important for small solvent molecules far away from $T_{\mathrm{g}}$. From eq 3 


$$
E=R_{\mathrm{g}} T^{2} \frac{\partial\left[\ln \left(D_{\mathrm{p}}\right)\right]}{\partial T}
$$

Using eq 5 in conjunction with the derivative of eq 4 with respect to temperature, we can derive the activation energy from the free-volume theory, $E_{\mathrm{FV}}$, as

$$
E_{\mathrm{FV}}=\frac{\gamma \hat{V}_{2}^{*} \xi R_{\mathrm{g}} T^{2}}{K_{12}\left(K_{22}-T_{\mathrm{g}^{2}}+T\right)^{2}}
$$

Because free-volume parameters are available for $\mathrm{PBD},{ }^{6}$ we can compare the activation energy obtained using the free-volume theory and the correlation of experimental data. From eq 6 , we observe that the activation energy is not a constant but a temperature-dependent quantity. However, if $T \gg K_{22}-T_{g 2}, E_{F V} \rightarrow R_{g} \gamma \hat{V}_{2}^{*} \xi / K_{12}$, a constant. For the case of PBD, although the activation energy is temperature dependent, an average activation energy in the temperature range of interest can be calculated by the integration of eq 6 .

We look to the equivalence of the Arrhenius and free-volume approaches to relate $D_{\mathrm{o}}$ and $\mathrm{E}_{\mathrm{Arr}}$. Substituting the result of eq 6 in eq 4, we obtain

$$
\ln \left(D_{\mathrm{p}}\right)=\ln \left(\tilde{D}_{\mathrm{o}}\right)-\frac{E_{\mathrm{FV}}}{R_{\mathrm{g}} T}\left(\frac{K_{22}-T_{\mathrm{g}^{2}}+T}{T}\right)
$$

Equating eqs 3 and 7 and recognizing that $E_{\text {Arr }}$ $=E_{\mathrm{FV}}=E$ yields

$$
\ln \left(D_{\mathrm{o}}\right)=\ln \left(\tilde{D}_{\mathrm{o}}\right)-\frac{E}{R_{\mathrm{g}} T}\left(\frac{K_{22}-T_{\mathrm{g}^{2}}}{T}\right)
$$

From eq 8, it is clear that $D_{\mathrm{o}}$ and $E$ are related for a given solvent diffusing in a polymer. Zhurkov and Ryskin ${ }^{12}$ have also shown that when Arrhenius behavior is observed for diffusion in polymersolvent systems, there is a correlation between the $D_{\mathrm{o}}$ term and the activation energy. The activation energy is proportional to the size of the penetrant molecule. The activation energy correlated using the Arrhenius approach is an average value for the temperature range of interest. It would be interesting to see if the pre-exponential factor is related to $E$ for different solvents diffusing in the same polymer over a similar temperature range. The second term on the right-hand side of eq 8 is temperature dependent, whereas both $D_{\mathrm{o}}$ and $\tilde{D}_{\mathrm{o}}$ are considered to be temperature independent. This is not a problem because the Arrhenius approach is in principle valid over a relatively narrow temperature range. In this case the second term on the right-hand side of eq 8 is approximately constant, or it is valid at temperatures where $T \gg K_{22}-T_{\mathrm{g} 2}$. Data correlated over different temperature ranges yield different average values of $D_{\mathrm{o}}$ and $E$. In practice, the Arrhenius approach is used over a relatively narrow range of temperatures to obtain a reasonable description of data within experimental scatter. Further, from eq 8 it is easy to see that at high temperatures (which also corresponds to small activation energy), the pre-exponential factor for the Arrhenius approach and the free-volume approach become the same. A significant advantage of using the free-volume theory to obtain the activation energy is that one could potentially predict average activation energies and thus different $D_{0}$ values for different temperature ranges.

\section{Prediction of the Partitioning of the Solvent}

Lee and Danner ${ }^{13}$ made a comparison of methods for predicting the infinite dilution solvent-activity coefficients in polymer solutions. They found that the group contribution, lattice-fluid equation-ofstate (GCLF-EoS) and the UNIFAC free-volume (UNIFAC-FV) models were generally the best. On the basis of these results, the data collected in this study were compared with the prediction from these two models.

The GCLF-EoS is a group contribution formulation based on the equation of state of Panayiotou and Vera ${ }^{14}$

$$
\frac{\tilde{p}}{\tilde{T}}=\ln \left(\frac{\tilde{v}}{\tilde{v}-1}\right)+\frac{z}{2} \ln \left(\frac{\tilde{v}+q / r-1}{\tilde{v}}\right)-\frac{\theta^{2}}{\tilde{T}}
$$

where $\tilde{p}, \tilde{T}$, and $\tilde{v}$ are the reduced pressure, temperature, and molar volume respectively

$$
\begin{aligned}
\tilde{p}=P / P^{*} \quad \tilde{T}=T / T^{*} \quad \tilde{v} & =v / v^{*} \\
\theta & =\frac{q / r}{\tilde{v}+q / r-1}
\end{aligned}
$$

and

$$
P^{*}=z \varepsilon^{* / 2} v_{\mathrm{h}} \quad T^{*}=z \varepsilon^{* / 2} R_{\mathrm{g}} \quad v^{*}=v_{\mathrm{h}} r
$$

with the following mixing rules: 


$$
r=x_{1} r_{1}+x_{2} r_{2} \quad q=x_{1} q_{1}+x_{2} q_{2} \quad v^{*}=x_{1} v_{1}^{*}+x_{2} v_{2}^{*}
$$

$$
\begin{aligned}
& \varepsilon^{*}=\theta_{1} \varepsilon_{1}^{*}+\theta_{2} \varepsilon_{2}^{*}-\theta_{1} \theta_{2} \Gamma_{12} \Delta \varepsilon \\
& \Delta \varepsilon=\varepsilon_{11}^{*}+\varepsilon_{22}^{*}-2 \varepsilon_{12}^{*} \quad \varepsilon_{\mathrm{ij}}=\sqrt{\varepsilon_{\mathrm{ii}} \varepsilon_{\mathrm{ij}}}\left(1-k_{\mathrm{ij}}\right)
\end{aligned}
$$

The molecular interaction parameter $\epsilon^{*}$ is a function of the pure component interaction energy, $\epsilon_{\mathrm{ii}}$, and the cross interaction energy, $\epsilon_{\mathrm{ij}} . k_{\mathrm{ij}}$ is the molecular interaction parameter. More details about this equation may be obtained in the original article of Panayiotou and Vera ${ }^{14}$ and about the group contribution implementation in the article of Lee and Danner. ${ }^{15}$

Oishi and Prausnitz ${ }^{16}$ modified the highly successful UNIFAC model of Fredenslund et al. ${ }^{17}$ to include a contribution for free-volume differences between the polymer and solvent molecules. The free-volume term contribution derived from the Flory equation of state $^{18}$ was added to the UNIFAC model to arrive at an expression for the weight-fraction activity coefficient of a solvent in a polymer as follows

$$
\ln \Omega_{1}=\ln \frac{a_{1}}{w_{1}}=\ln \Omega_{1}^{\mathrm{C}}+\ln \Omega_{1}^{\mathrm{R}}+\ln \Omega_{1}^{\mathrm{FV}}
$$

The combinatorial and residual contributions, $\Omega_{1}^{\mathrm{C}}$ and $\Omega_{1}^{\mathrm{R}}$, are identical to the original UNIFAC terms. The free-volume contribution is given by

$$
\ln \Omega_{1}^{\mathrm{FV}}=3 C_{1} \ln \left[\frac{\tilde{v}_{1}^{1 / 3}-1}{\tilde{v}_{\mathrm{m}}^{1 / 3}-1}\right]-C_{1}\left[\frac{\tilde{v}_{1}}{\tilde{v}_{\mathrm{m}}}-1\right]\left[\frac{\tilde{v}_{1}^{1 / 3}}{\tilde{v}_{1}^{1 / 3}-1}\right]
$$

Here $C_{1}$ is an external degree of freedom parameter for the solvent.

\section{EXPERIMENTAL}

\section{Apparatus}

The mutual diffusion coefficients for PBD solvent systems were measured using the IGC technique. Hadj Romdhane and Danner ${ }^{2}$ reported the detailed experimental procedure. In brief, the chromatograph used in this work was a Varian 3400 equipped with a flame-ionization detector (FID), an on-column injector, and an air-circulating oven. Ultra-high-purity helium was used in all experiments as the carrier gas. Dry air and highpurity hydrogen were used to produce the flame in the FID. Methane was used as the marker gas. A makeup stream that bypasses the column was used to provide an adequate flow to the detector and reduce mixing effects by pushing the column gas into the detector at high velocities. The temperature of the injection block and the detector assembly were set about $50{ }^{\circ} \mathrm{C}$ above the column temperature to avoid condensation.

\section{Materials}

The solvents (purity $>99 \%$ ) were obtained from Aldrich Chemical and used without further purification. One sample of PBD was obtained from the American Polymer Standards Corp. (APSC). It had a weight-average molecular weight of 240,000 , a polydispersity of 2.18 , and a structure of $55 \%$ trans- 1,$4 ; 36 \%$ cis-1,4; and 9\% 1,2-addition. A quartz capillary column was prepared by the Restek Co. (Bellefonte, PA). The column was $8.4 \mathrm{~m}$ long with an inner diameter of $530 \mu \mathrm{m}$ and a polymer coating of $5 \mu \mathrm{m}$.

In the study of the PBD-ethylbenzene system, a PBD sample obtained from the B. F. Goodrich Co. in 1993 was used to compare data from two different sources. The PBD was specified as $95 \%$ cis-1,4 and had a Mooney viscosity of 38-46. A capillary column with an inner diameter of 530 $\mu \mathrm{m}$ and length $12 \mathrm{~m}$ was made with it by Supelco, Inc. (Bellefonte, PA). The thickness of the polymer coating was $2 \mu \mathrm{m}$. This column had previously been used to run infinite dilute IGC experiments on PBD-ethylbenzene in 1993. Because all experiments were done well above the $T_{\mathrm{g}}$ of the PBD $\left(-95^{\circ} \mathrm{C}\right)$, PBDwas in rubbery form.

\section{RESULTS AND DISCUSSION}

\section{PBD-Ethylbenzene System}

Using ethylbenzene with the APSC sample, diffusivities, $D_{\mathrm{p}}$, and partition coefficients, $K$, were obtained between 50 and $125{ }^{\circ} \mathrm{C}$. Three determinations were made for each temperature. Figure 1 shows a typical comparison of the elution profile and CCIGC model for the experiment at $70{ }^{\circ} \mathrm{C}$. The curve obtained from the CCIGC model fits the data well. Data for the B. F. Goodrich sample were collected between 20 and $70{ }^{\circ} \mathrm{C}$. The averages of three independent measurements for the partition and diffusion coefficients for ethylben- 


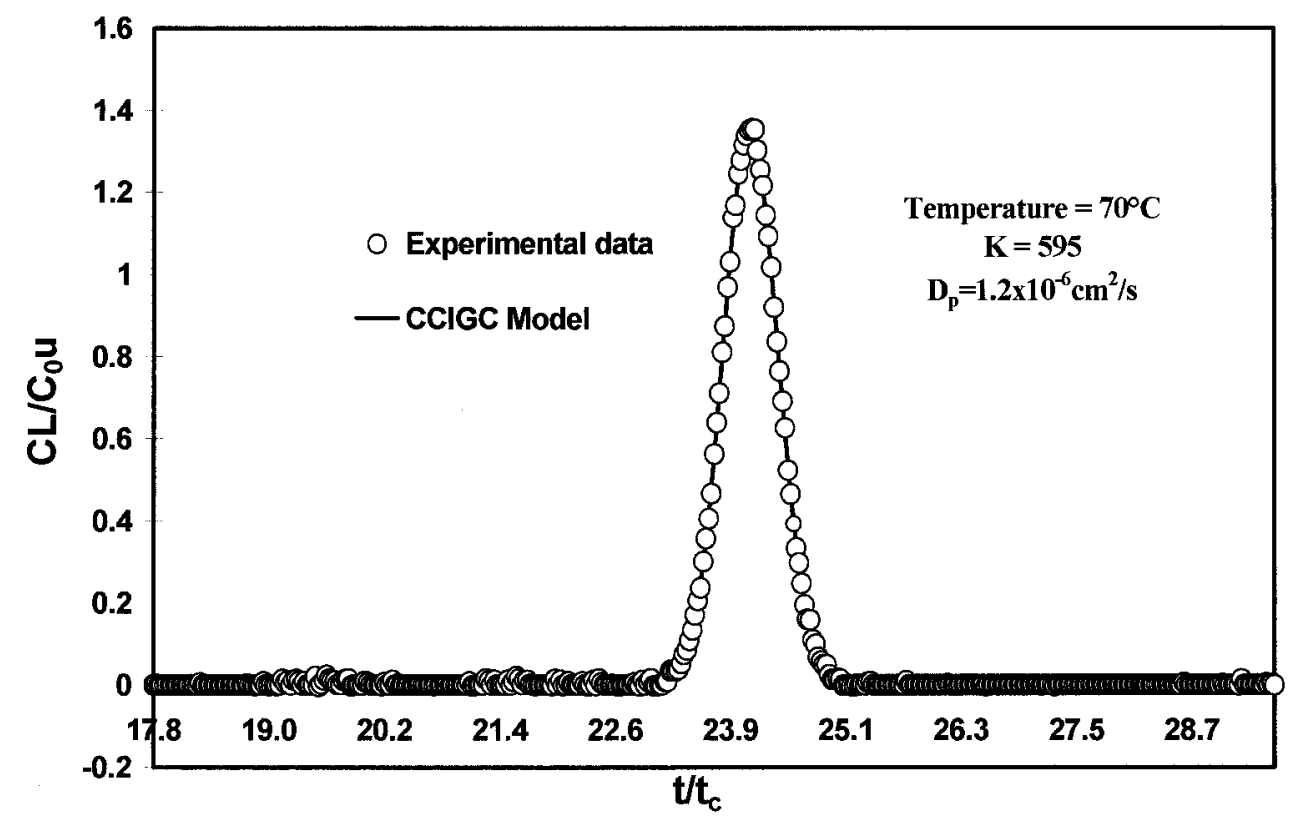

Figure 1. Comparison between the experimental and model elution profiles for the ethylbenzene/PBD system.

zene in both PBD samples are listed in Table 1. The deviations between these measurements were generally less than $1 \%$. At corresponding temperatures the partition coefficients of the two different PBD samples agree almost exactly. Figure 2 demonstrates the variations of the infinitely dilute partition coefficients as functions of inverse temperature. Clearly, $\ln (K)$ shows a linear dependence upon the inverse absolute temperature.
The predictions of WFAC at infinite dilution, $\Omega^{\infty}$, obtained with the UNIFAC-FV and GCLFEoS models are depicted in Figure 3. Both models predict results that are too low, with the UNIFAC-FV being somewhat better. These predictions, however, are better than the scatter in results frequently found when comparing data from different sources as shown by Lee and Danner. ${ }^{13}$

Table 1. Comparison of the Data Taken with the Goodrich and APSC Polymer for the PBD-Ethylbenzene System

\begin{tabular}{|c|c|c|c|c|}
\hline \multirow[b]{2}{*}{$T\left({ }^{\circ} \mathrm{C}\right)$} & \multicolumn{2}{|c|}{$K$} & \multicolumn{2}{|c|}{$D_{\mathrm{p}}\left(\mathrm{cm}^{2} / \mathrm{s}\right)$} \\
\hline & $\begin{array}{l}\text { Goodrich } \\
\text { PBD }\end{array}$ & $\begin{array}{l}\text { APSC } \\
\text { PBD }\end{array}$ & $\begin{array}{l}\text { Goodrich } \\
\text { PBD }\end{array}$ & $\begin{array}{l}\text { APSC } \\
\text { PBD }\end{array}$ \\
\hline 20 & 6360 & & $5.5 \times 10^{-8}$ & \\
\hline 30 & 3670 & & $1.9 \times 10^{-7}$ & \\
\hline 40 & 2180 & & $3.5 \times 10^{-7}$ & \\
\hline 50 & 1400 & 1420 & $4.9 \times 10^{-7}$ & $5.6 \times 10^{-7}$ \\
\hline 60 & 908 & 899 & $5.2 \times 10^{-7}$ & $9.3 \times 10^{-7}$ \\
\hline 70 & 617 & 595 & $6.8 \times 10^{-7}$ & $1.2 \times 10^{-6}$ \\
\hline 80 & & 408 & & $1.8 \times 10^{-6}$ \\
\hline 90 & & 286 & & $2.2 \times 10^{-6}$ \\
\hline 100 & & 207 & & $2.4 \times 10^{-6}$ \\
\hline 110 & & 152 & & $2.7 \times 10^{-6}$ \\
\hline 120 & & 114 & & $2.9 \times 10^{-6}$ \\
\hline 125 & & 101 & & $3.5 \times 10^{-6}$ \\
\hline
\end{tabular}




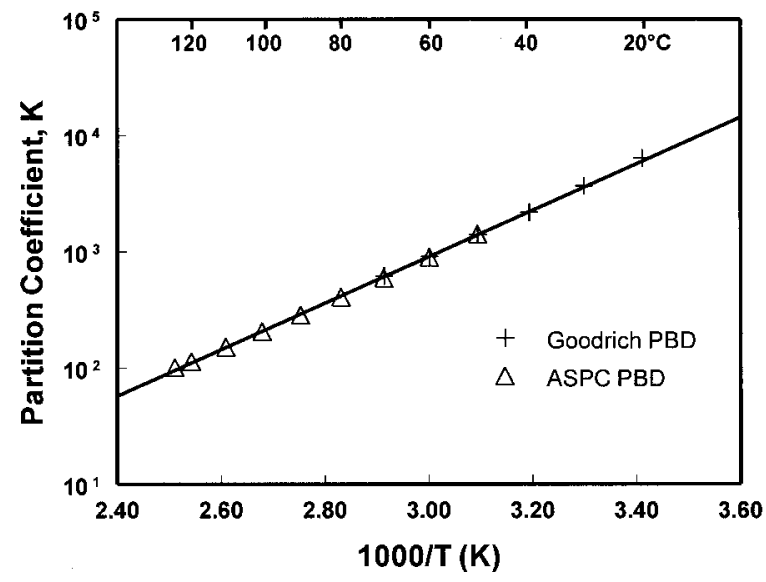

Figure 2. Partition coefficients of ethylbenzene in PBD.

Figure 4 illustrates the comparison in the diffusion coefficients obtained from the B. F. Goodrich polymer and the APSC polymer. The two sets of data are in relatively good agreement with the exception of the value at $20^{\circ} \mathrm{C}$. Overall, the diffusion coefficients are consistent and increase with temperature. The data were correlated with the Arrhenius function (eq 3). The $D_{\text {o }}$ and $E$ values obtained are listed in Table 2.

\section{PBD-Acrylonitrile and PBD-Styrene Systems}

The infinitely dilute, acrylonitrile data were obtained using the PBD column containing the sample from APSC. $K$ and $D_{\mathrm{p}}$ values were obtained from 50 to $125^{\circ} \mathrm{C} . K$ and $D_{\mathrm{p}}$ values listed in Table

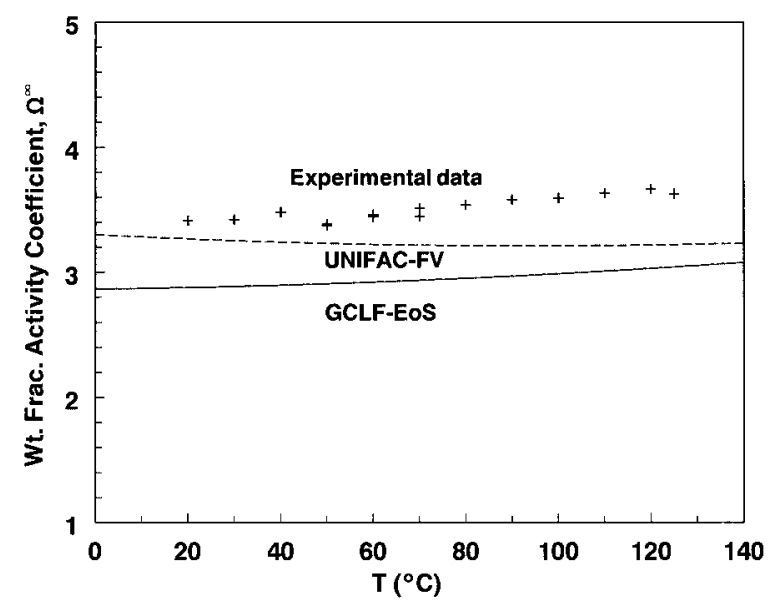

Figure 3. Comparison of experimental weight-fraction activity coefficients with predictions from the group contribution theories for the ethylbenzene/PBD system.

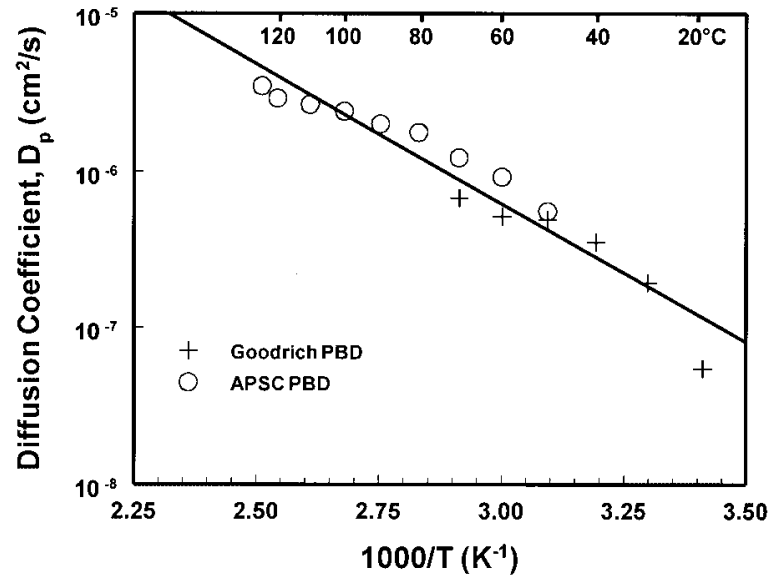

Figure 4. Diffusion coefficients of ethylbenzene in PBD with Arrhenius correlation.

3 are the average of three independent measurements that varied by less than $1 \%$. Comparisons of the CCIGC model and the experimental elution curves for this system indicated similar agreement as shown in Figure 1 for the PBD-ethylbenzene system. Table 3 includes the partition and diffusion coefficients for the PBD (APSC)-styrene system. Experiments in this case were performed from 50 to $100{ }^{\circ} \mathrm{C}$ at $10{ }^{\circ} \mathrm{C}$ intervals. Figure 5 portrays the influence of the temperature on the partition coefficients for these two solvents. The partition coefficients for the acrylonitrile range from approximately 7 to 40 , values that are much lower than those of styrene (ca. 280-2100). This demonstrates that PBD is more soluble in styrene than in acrylonitrile. Predictions based on the GCLF-EoS and UNIFAC FV could be made for the styrene-PBD system (Fig. 6). As in the case of ethylbenzene, the UNIFAC-FV method is somewhat better, but both models predict values that are somewhat low. Values for acrylonitrile could not be obtained because some group parameters were missing.

As depicted in Figure 7, the styrene diffusivities have stronger temperature dependencies than those of acrylonitrile in PBD. In the temperature range from 50 to $100{ }^{\circ} \mathrm{C}$, the diffusion coefficient of styrene in PBD changes from $2 \times 10^{-6}$ to $5 \times 10^{-7} \mathrm{~cm}^{2} / \mathrm{s}$, whereas the change for acrylonitrile is only from $1.4 \times 10^{-6}$ to $3 \times 10^{-6} \mathrm{~cm}^{2} / \mathrm{s}$. The diffusivity data also have been correlated with the Arrhenius function (eq 3). The $D_{\mathrm{o}}$ and $E$ values obtained are included in Table 2.

There are a few studies where the diffusion of solvents in PBD have been measured. ${ }^{6,7,19}$ Guillermo et al. ${ }^{7}$ examined diffusion of cyclohexane in 
Table 2. Arrhenius Parameters for Polybutadiene Solvent Systems

\begin{tabular}{clccc}
\hline \multirow{2}{*}{ Source } & \multicolumn{1}{c}{ Solvent } & Occupied Volume $(0 \mathrm{~K})$ & $D_{\mathrm{o}}\left(\mathrm{cm}^{2} / \mathrm{s}\right)$ & $E(\mathrm{~kJ} / \mathrm{mol})$ \\
\hline \multirow{2}{*}{ This study } & Acrylonitrile & 47.58 & $4.01 \times 10^{-5}$ & 8.41 \\
& Ethylbenzene & 100.43 & $1.54 \times 10^{-1}$ & 34.29 \\
Faridi et al. $^{6}$ & Styrene & 93.70 & $6.27 \times 10^{-2}$ & 30.71 \\
& Chloroform & 60.89 & $1.97 \times 10^{-3}$ & 20.13 \\
& Cyclohexane & 87.36 & $3.91 \times 10^{-3}$ & 23.16 \\
& MEK & 73.20 & $8.30 \times 10^{-3}$ & 23.80 \\
& Heptane & 111.75 & $1.69 \times 10^{-2}$ & 24.94 \\
& Toluene & 84.48 & $1.81 \times 10^{-2}$ & 25.79 \\
\hline
\end{tabular}

PBD at different solvent concentrations using NMR techniques. Although they have not measured diffusivity of trace amounts of cyclohexane, their correlations using the free-volume theory are in excellent agreement with the data of Faridi et al. ${ }^{6}$ Brown et al. ${ }^{19}$ used limiting slope data from a desorption experiment to determine diffusion coefficients at zero concentration. Although diffusion coefficients can be estimated by this method, in practice scatter in the limiting slope data significantly affects the values at zero concentration. Because these data did not agree with those reported in other studies, ${ }^{6,7}$ we did not use the data reported by Brown et al. ${ }^{19}$

Using the data obtained in this study and by Faridi et al., ${ }^{6}$ the pre-exponential factor and activation energy for solvent diffusion were correlated using the Arrhenius approach described in a previous section (eq 3). Figure 8 describes the correlation between the pre-exponential factor $D_{\mathrm{o}}$ and $E$ for the various solvents diffusing in PBD. The correlation confirms that $D_{\mathrm{o}}$ and $E$ are indeed related for different solvents diffusing in the same polymer. The advantage of using an activation energy specific to the temperature range being considered is that one can obtain better estimates of diffusivity in that temperature range. The activation energy was also calculated according to eq 6. Once $\xi$ is known for one solvent diffusing in a polymer, all other $\xi$ values can be calculated if the molar volume of the solvent at $0 \mathrm{~K}$ is known (occupied volume). The molar volume at $0 \mathrm{~K}$ was obtained from a previous study ${ }^{20}$ or calculated using group contribution techniques. ${ }^{21,22}$ The $\xi$ for the toluene-PBD system was obtained from Faridi et al. ${ }^{6}$ From Table 4 we observe that over a temperature range of $100 \mathrm{~K}$ for ethylbenzene diffusing in $\mathrm{PBD}$, the activation energy calculated using the free-volume approach varies by a factor of approximately 2. Nevertheless, the data were adequately described by a single value of activation energy.

The relationships between the pre-exponential factor and activation energy with the occupied volume are depicted in Figure 9. Although the number of data points is admittedly small, the correlations appear to be rather good, and the trends agree with previous observations. ${ }^{23}$ Figures 8 and 9 can be used to predict the diffusivity of other solvents in PBD on the basis of only the

Table 3. Partition and Diffusion Coefficients for PBD Systems

\begin{tabular}{|c|c|c|c|c|}
\hline \multirow[b]{2}{*}{$T\left({ }^{\circ} \mathrm{C}\right)$} & \multicolumn{2}{|c|}{ PBD-Styrene } & \multicolumn{2}{|c|}{ PBD-Acrylonitrile } \\
\hline & $K$ & $D_{\mathrm{p}}\left(\mathrm{cm}^{2} / \mathrm{s}\right)$ & $K$ & $D_{\mathrm{p}}\left(\mathrm{cm}^{2} / \mathrm{s}\right)$ \\
\hline 50 & 2070 & $6.1 \times 10^{-7}$ & 39.6 & $1.6 \times 10^{-6}$ \\
\hline 60 & 1300 & $9.4 \times 10^{-7}$ & & \\
\hline 70 & 845 & $1.3 \times 10^{-6}$ & 22.5 & $2.2 \times 10^{-6}$ \\
\hline 80 & 576 & $1.7 \times 10^{-6}$ & & \\
\hline 90 & 400 & $2.3 \times 10^{-6}$ & 14.8 & $2.7 \times 10^{-6}$ \\
\hline 100 & 284 & $2.9 \times 10^{-6}$ & & \\
\hline 110 & & & 10.2 & $2.7 \times 10^{-6}$ \\
\hline 125 & & & 6.9 & $3.0 \times 10^{-6}$ \\
\hline
\end{tabular}




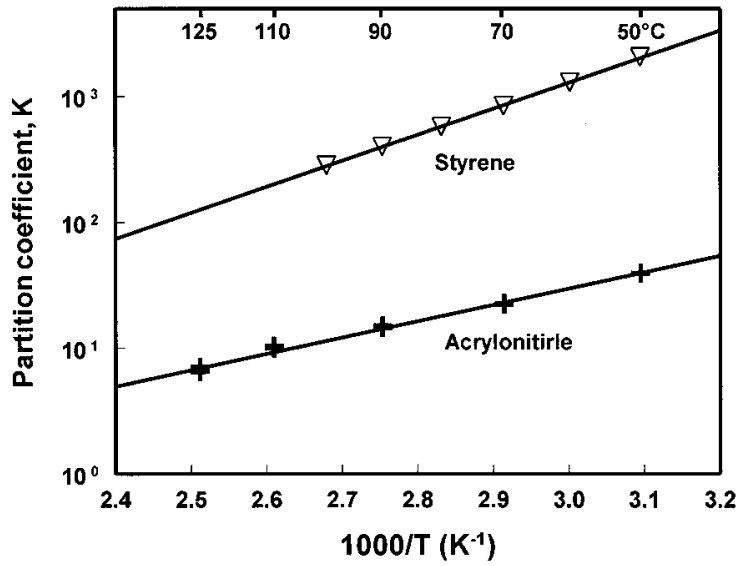

Figure 5. Partition coefficients of styrene and acrylonitrile in PBD.

size of the solvent. Heptane was excluded from this correlation because previous studies indicated that long-chain hydrocarbon molecules such as heptane do not jump as one unit. ${ }^{24}$ It is encouraging that good correlations of the activation energy and pre-exponential terms with the molar volume of the solvent at $0 \mathrm{~K}$ were obtained. The agreement between the calculated and predicted activation energies is excellent for most solvents with the exception of cyclohexane. It is unclear why there should be a discrepancy for cyclohexane; it may be due to experimental scatter.

In Figure 10, the correlations of activation energies obtained from the free-volume theory $\left(E_{\mathrm{FV}}\right)$ and experimental data $\left(E_{\mathrm{Arr}}\right)$ are shown. Vrentas et al. ${ }^{25}$ suggest that a correction should be used for linear molecules such as heptane because they

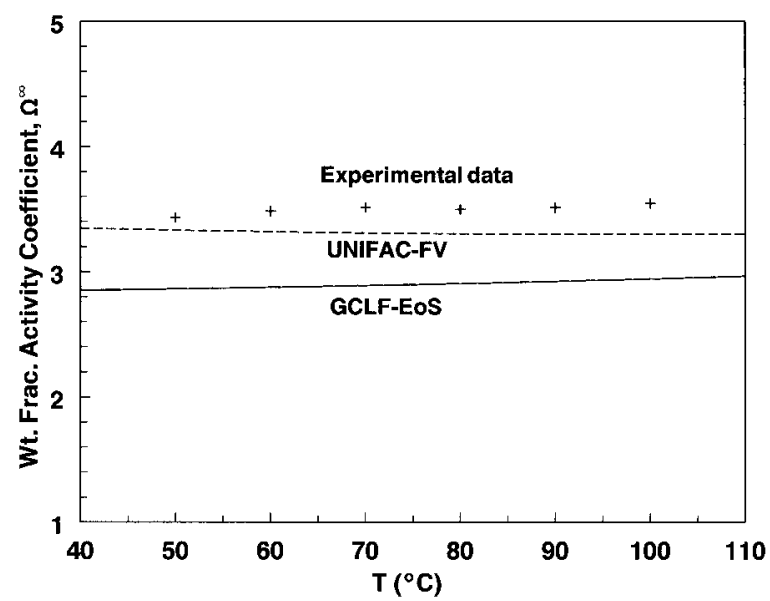

Figure 6. Weight-fraction activity coefficients of styrene in $\mathrm{PBD}$

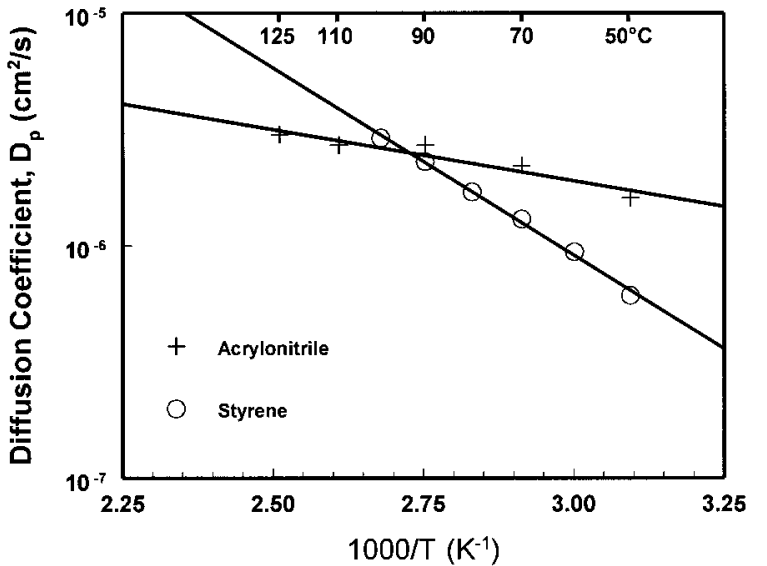

Figure 7. Diffusion coefficients of styrene and acrylonitrile in PBD.

do not diffuse as one unit. The activation energy for heptane has been corrected on the basis of the preceding method.

Jiang and $\mathrm{Han}^{26}$ explored the idea that data from one polymer-solvent system can be used to predict diffusion for other solvents in the same polymer. They assumed that the ratio of the jumping unit size to the critical temperature of the solvent, $\xi / T_{\mathrm{c}}$, was constant for all solvents in a given polymer-solvent system. The solid symbols and line in Figure 11 indicate their data and the proposed correlation. The correlation is clearly not valid for a large number of solvents. Other solvents show significant scatter around the line.

\section{CONCLUSIONS}

Diffusion and partition coefficient data for ethylbenzene, acrylonitrile, and styrene in PBD over a

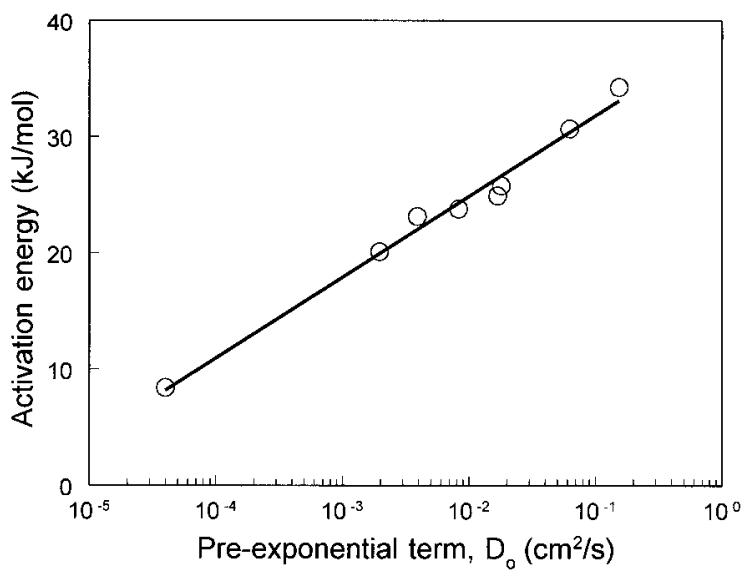

Figure 8. Relationship between the Arrhenius preexponential term and the activation energy. 
Table 4. Activation Energy as a Function of Temperature Calculated Using the Free-Volume Theory

\begin{tabular}{rc}
\hline$T\left({ }^{\circ} \mathrm{C}\right)$ & $E_{\mathrm{FV}}(\mathrm{kJ} / \mathrm{mol})$ \\
\hline 20 & 38.04 \\
30 & 35.95 \\
40 & 33.86 \\
50 & 32.19 \\
60 & 30.93 \\
70 & 29.68 \\
80 & 28.42 \\
90 & 27.59 \\
100 & 26.75 \\
110 & 25.92 \\
120 & 25.08 \\
\hline
\end{tabular}

temperature range from 50 to $125{ }^{\circ} \mathrm{C}$ were successfully determined by the IGC method. On the basis of the behavior of the data as a whole, it is concluded that the CCIGC model provides an adequate description of peak retention and dispersion for the PBD-solvent systems. The UNIFACFV model gives somewhat better predictions than GCLF-EoS of the infinite dilute weight-fraction activity coefficients, but both consistently give values that are somewhat low. The diffusivity data were correlated well with a standard Arrhenius equation, and the regression parameters were related to each other and to the occupied volume.

The correlations developed herein with some limited data for a polymer-solvent system can be used to predict diffusion of other solvents in the

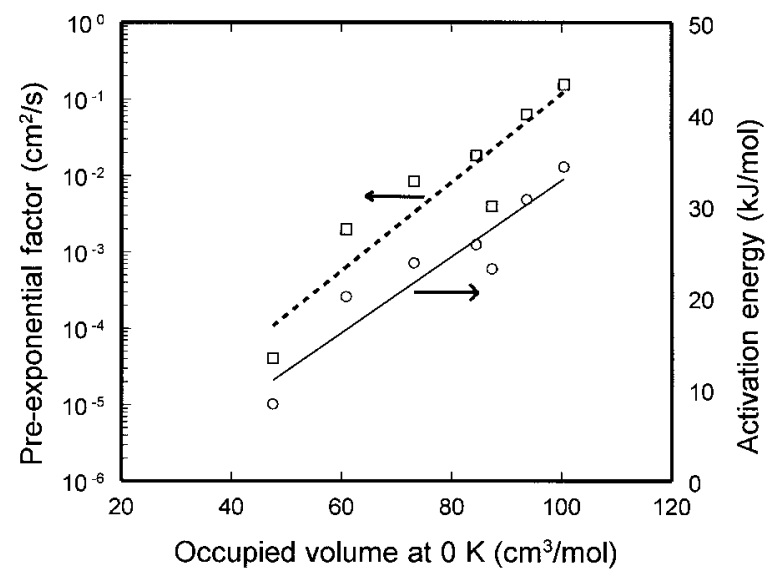

Figure 9. Correlation of the pre-exponential factor and activation energy with molar volume at $0 \mathrm{~K}$.

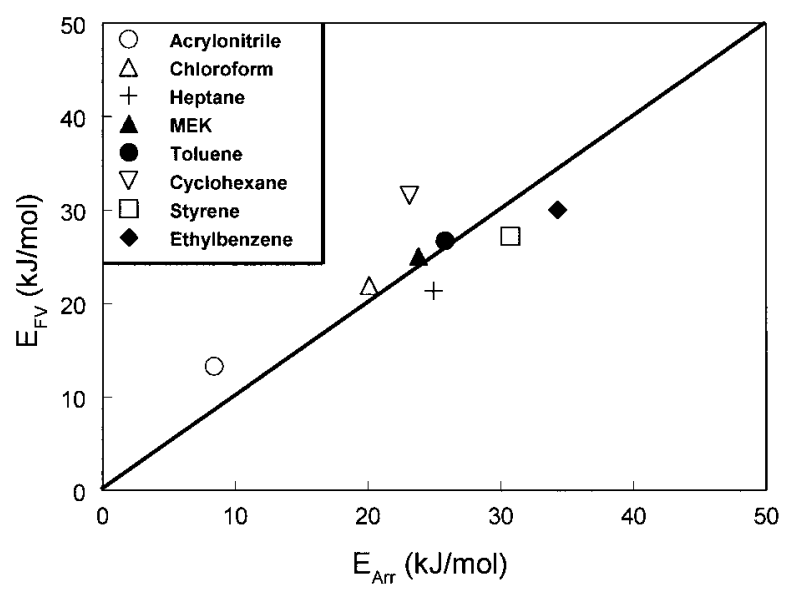

Figure 10. Correlation of activation energies obtained from the Arrhenius and free-volume models.

same polymer. Further, the results of this study indicated that there is a strong correlation between the value of the pre-exponential factor and the activation energy. Although the activation energy varies even over a small temperature range, the data can be correlated well with a constant value of the activation energy. For most of the solvents considered, the predicted average activation energies and correlated activation energies reasonably agree with one another. Consequently, in the absence of any data, a reasonable estimate of diffusion of a solvent in a polymer may be obtained given the diffusion of other solvents in the same polymer at temperatures above the $T_{\mathrm{g}}$ 's of the polymer.

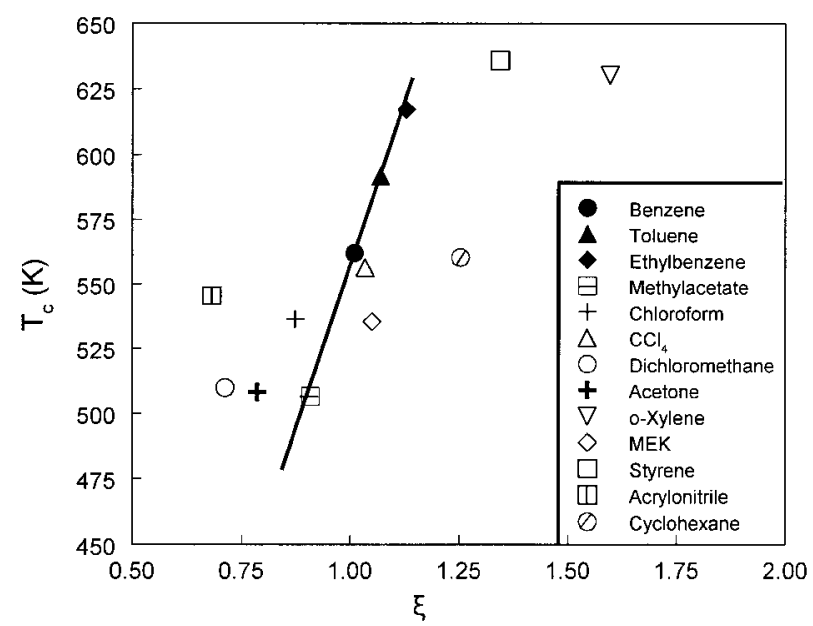

Figure 11. Correlation of the ratio of the jumping unit size and the critical temperature. The solid symbols and line represent the correlation suggested by Jiang and Han. ${ }^{26}$ 
The authors gratefully acknowledge DSM Research (Geleen, Netherlands) for the financial support of this work.

\section{NOMENCLATURE}

\begin{tabular}{|c|c|}
\hline$A$ & Activity \\
\hline$C$ & $\begin{array}{l}\text { Area average solute concentration in the } \\
\text { gas phase }\left(\mathrm{mol} / \mathrm{cm}^{3}\right)\end{array}$ \\
\hline$C^{\prime}$ & $\begin{array}{l}\text { Solute concentration in the polymer phase } \\
\left(\mathrm{mol} / \mathrm{cm}^{3}\right)\end{array}$ \\
\hline$C_{1}$ & External degree of freedom parameter \\
\hline$c_{0}$ & Strength of the inlet impulse $\left(\mathrm{mol}-\mathrm{s} / \mathrm{cm}^{3}\right)$ \\
\hline$D$ & $\begin{array}{l}\text { Solvent diffusion coefficient in the poly- } \\
\text { mer phase }\left(\mathrm{cm}^{2} / \mathrm{s}\right)\end{array}$ \\
\hline$D_{\mathrm{g}}$ & $\begin{array}{l}\text { Solvent diffusion coefficient in the mobile } \\
\text { phase }\left(\mathrm{cm}^{2} / \mathrm{s}\right)\end{array}$ \\
\hline$D_{\sim}$ & Arrhenius pre-exponential factor $\left(\mathrm{cm}^{2} / \mathrm{s}\right)$ \\
\hline$\tilde{D}_{\mathrm{o}}$ & $\begin{array}{l}\text { Free-volume theory pre-exponential factor } \\
\left(\mathrm{cm}^{2} / \mathrm{s}\right)\end{array}$ \\
\hline$E$ & $\begin{array}{l}\text { Overall activation energy for diffusion ob- } \\
\text { tained using the Arrhenius expression } \\
(\mathrm{kJ} / \mathrm{mol})\end{array}$ \\
\hline$E_{\mathrm{FV}}$ & $\begin{array}{l}\text { Overall activation energy for diffusion ob- } \\
\text { tained using the free-volume theory (kJ/ } \\
\text { mol) }\end{array}$ \\
\hline K & Equilibrium partition coefficient \\
\hline$K_{11} / \gamma$ & Free-v \\
\hline$K_{22}$ & ume parameter \\
\hline$k_{\mathrm{ij}}$ & Molecular interaction parameter \\
\hline$L$ & Length of the column $(\mathrm{cm})$ \\
\hline$P$ & Pressure $(\mathrm{Pa})$ \\
\hline$q$ & Surfa \\
\hline$R$ & Radius of the polymer-gas interface $(\mathrm{cm})$ \\
\hline$R_{\mathrm{g}}$ & Gas constant (J/mol-K) \\
\hline$r$ & Number of segments in the molecule \\
\hline$s$ & Laplace operator \\
\hline$T$ & Temperature (K) \\
\hline$T_{\mathrm{gi}}$ & $\begin{array}{l}\text { Glass-transition temperature of compo- } \\
\text { nent } \mathrm{i}(\mathrm{K})\end{array}$ \\
\hline$u$ & Mean velocity of the carrier gas $(\mathrm{cm} / \mathrm{s})$ \\
\hline$v_{\mathrm{h}}$ & $\begin{array}{l}\text { Molar volume of a lattice site }(=9.75 \\
\left.\times 10^{-3} \mathrm{~m}^{3} / \mathrm{mol}\right)\end{array}$ \\
\hline$x_{\mathrm{i}}$ & Mole fraction of component $\mathrm{i}$ \\
\hline & Lattice coordination number $(=10)$ \\
\hline
\end{tabular}

\section{Greek Letters}

$\Gamma_{\mathrm{ij}}$ Nonrandomness factor

$\epsilon^{*} \quad$ Characteristic interaction parameter

$\theta_{i} \quad$ Surface-area fraction of component $i$

$\xi \quad$ Ratio of the molar volume of the jumping unit of the solvent to that of the polymer $\tau \quad$ Film thickness in the capillary column $(\mathrm{cm})$

$\Omega_{\mathrm{i}}$ Weight-fraction activity coefficient of component i

$\omega_{\mathrm{i}} \quad$ Weight fraction of component $\mathrm{i}$

\section{REFERENCES AND NOTES}

1. Arnould, D. D.; Laurence, R. L. Solute Diffusion in Polymers by Capillary Column Inverse Gas Chromatography. In Inverse Gas Chromatography; Lloyd, D. R.; Ward, T. C.; Shreiber, H. P., Eds.; ACS Symposium Series 391; American Chemical Society: Washington, DC, 1989; p 87.

2. Hadj Romdhane, I.; Danner, R. P. AIChE J 1993, 39, 625 .

3. Hadj Romdhane, I.; Danner, R. P.; Duda, J. L. Ind Eng Chem Res 1995, 34, 2536.

4. Vrentas, J. S.; Duda, J. L. J Polym Sci Polym Phys Ed 1977, 15, 403.

5. Vrentas, J. S.; Duda, J. L. J Polym Sci Polym Phys Ed 1977, 15, 417.

6. Faridi, N.; Duda, J. L.; Danner, R. P. Rubber Chem Technol 1996, 69(2), 234.

7. Guillermo, A.; Todica, M.; Cohen-Addad, J. P. Macromolecules 1993, 26, 3946.

8. Brown, W. R.; Jenkins, R. B.; Park, G. S. J Polym Sci Polym Symp 1973, 41, 45.

9. Chalykh, A. E.; Bobkov, A. S. Polym Sci USSR 1990, 10, 2033.

10. Hong, S.-U. J Appl Polym Sci 1996, 61, 833.

11. Pawlisch, C. A.; Macris, A.; Laurence, R. L. Macromolecules 1987, 20, 1564.

12. Zhurkov, S. N.; Ryskin, G. Y. J Technol Phys USSR 1954, 24, 797.

13. Lee, B.-C.; Danner, R. P. Fluid Phase Equilib 1997, 128, 97.

14. Panayiotou, C.; Vera, J. H. Polym J 1982, 14, 681.

15. Lee, B.-C.; Danner, R. P. AIChE J 1996, 42, 837.

16. Oishi, T.; Prausnitz, J. M. Ind Eng Chem Res Dev 1978, 17, 333.

17. Fredenslund, Aa.; Jones, R. L.; Prausnitz, J. M. AIChE J 1975, 21, 1086.

18. Flory, P. J.; Oriji, R. A.; Vrij, A. J Am Chem Soc 1964, 86, 3507.

19. Brown, W. R.; Jenkins, R. B.; Park, G. S. J Polym Sci Polym Symp 1973, 41, 45.

20. Zielinski, J. M.; Duda, J. L. AIChE J 1992, 38, 405.

21. Sugden, S. J Chem Soc 1927, 1786.

22. Biltz, W. Rauchemie der Festen Stoffe; Verlag von Leopold Voss: Leipzig, 1934; p 301.

23. Balashova, I. M.; Danner, R. P.; Puri, P. S.; Duda, J. L. Ind Eng Chem Res, 2001, 40, 3058.

24. Arnould, D.; Laurence, R. L. Ind Eng Chem Res 1992, 31, 218.

25. Vrentas, J. S.; Vrentas, C. M.; Faridi, N. Macromolecules 1996, 29, 3272.

26. Jiang, W. H.; Han, R. J Appl Polym Sci 2001, 80, 529. 